\title{
Characteristics of a Transactive Phenomenon in Relationships among Older Adults with Dementia, Nurses as Intermediaries, and Communication Robot
}

\author{
Kyoko Osaka1, Hiroko Sugimoto², Tetsuya Tanioka1, Yuko Yasuhara1, Rozzano Locsin', \\ Yueren Zhao ${ }^{3}$, Kikuko Okuda', Ken Saito ${ }^{1}$ \\ ${ }^{1}$ Department of Nursing, Institute of Biomedical Sciences, Tokushima University, Tokushima, Japan \\ ${ }^{2}$ Graduate School of Health Sciences, Tokushima University, Tokushima, Japan \\ ${ }^{3}$ Fujita Health University, Toyoake, Japan \\ Email: osaka@tokushima-u.ac.jp
}

How to cite this paper: Osaka, K., Sugimoto, H., Tanioka, T., Yasuhara, Y., Locsin, R., Zhao, Y.R., Okuda, K. and Saito, K. (2017) Characteristics of a Transactive Phenomenon in Relationships among Older Adults with Dementia, Nurses as Intermediaries, and Communication Robot. Intelligent Control and Automation, 8, 111-125. https://doi.org/10.4236/ica.2017.82009

Received: February 26, 2017

Accepted: May 23, 2017

Published: May 26, 2017

Copyright $\odot 2017$ by authors and Scientific Research Publishing Inc. This work is licensed under the Creative Commons Attribution International License (CC BY 4.0).

http://creativecommons.org/licenses/by/4.0/

\begin{abstract}
Healthcare for older adults with dementia is an important healthcare problem in Japan and in other developed countries. Importantly, healthcare workers in Japan are also getting older. In order to address solutions to this problem, using robots is being realized and is starting to assist healthcare and welfare practice needs. The aim of this study was to identify the characteristics of a transactive phenomenon in relationships among older adults with dementia, with nurses as intermediaries and with a cognitive skill-aid robot. Subjects were two institutionalized older adults who were diagnosed with dementia using Hasegawa's Dementia Scale-Revised; a Kabochan (Nodding Kabochan) fixed with a remote-controlled speaker, Pechat (Kabochan with Pechat). Measurements of autonomic nervous activity were done using wearable electrocardiography attached to the subjects for four hours. Heart Rate Variability data were assessed at various frequency bands using a HRV software tool. In a continuously recorded data, interbeat (R-R) intervals were obtained for a 1-min segment using the maximum entropy method. In this study, the two major spectral components of HRV, the variances of the low-frequency (LF: $0.04-0.15 \mathrm{~Hz}$ ) band and high-frequency (HF: $0.15-0.4 \mathrm{~Hz}$ ) band, were calculated. Activities and behaviors of subjects transacting with Kabochan were observed by the expert nurses and video-recorded. Throughout the transaction period, observations were recorded of patients' behavior, words uttered and over-all contents of the transactive conversation with the Kabochan or Kabochan with Pechat. Identified characteristics were transactive relationship phenomenon; changes in autonomic nervous system activity of persons with
\end{abstract}


dementia are expressed as per one minute, linguistic response of elderly people, transactive relations among Kabochan, Kabochan with Pechat, and nurses as intermediaries. These were exhibited as Figures: Situation 1, $A$ Sense of Fear, Situation 2, A Pleasant Sensation, and Situation 3, Misunderstanding and Confusion. It also showed that autonomic nervous system activity of persons with communication skills is actively changing in older adults with dementia. Conversation of adding Pechat to the Kabochan illustrated that nurses as intermediaries showed emotional conversations as established by effectively manipulating the Pechat. Also, it was revealed that if Kabochan with Pechat uttered words about patient's personal information in a conversation, the patient felt a sense of fear. The researchers suggest that when developing future communication robot for elderly with dementia, it is necessary to consider, for an effective conversation, transactive relationships including nurses as intermediaries. The role functions of these intermediaries have been shown of high versatility, indicating that situations such as these can also be controlled by other healthcare providers such as by occupational therapists.

\section{Keywords}

Transactive Phenomenon, Nursing as Intermediaries, Older Adults with Dementia, Communication Robot

\section{Introduction}

Challenges of a society with a decreasing population and increasing population of older adults with dementia are healthcare problems in Japan, and of other developing countries [1] [2]. In Japan, it is estimated that the number of patients with dementia will reach seven million by 2025, and 1 in 10 persons will be at risk of being afflicted with dementia [3]. The New Energy and Industrial Technology Development Organization (NEDO) launched a program called the Robot Care Equipment Development Partnership and served as the coordinating organization. In addition to matching services, NEDO provides various services including information sharing with government agencies, building networks between companies and researchers, and collecting feedback on future policies to promote the application of robotic nursing care devices [4]. With these initiatives, non-industrial robots were mass produced, examples of which were for those for walking support robots [5] [6] or communication robots [7] [8]. These robots are now being introduced in hospitals, nursing homes and in Japanese households.

In reviewing the literatures regarding elderly care, robots, and caring in nursing, new challenges regarding healthcare for the elderly population, it was found that care of the elderly population particularly those with dementia was emerging as a social issue, particularly on the aspect of sustaining adequate healthcare. Therefore, newer healthcare domains have to be considered, and innovation is found to be a required element. One of these innovations in Japan is using robots to assist in healthcare and nursing practice needs. Under this context, un- 
obtrusiveness is of paramount importance since the robot should be a natural participant of patients' daily life [9].

Animal-assisted interventions on elderly patients with dementia were found to have positive influences-reducing the degree of agitation and improving the degree and quality of social interaction. It was suggested that a substitute pet robot may yield encouraging results, but its use may require further investigation [10]. With this suggestion, robot therapy is considered another important intervention in the challenging health and innovative care practices needed in the care of elderly persons [11]. Nevertheless, two issues were realized regarding living with a human-type communication robot as a strategy; to improve cognitive functions and prevent cognitive decline in the elderly [12]. With robot therapy, it was found that only a few aspects of its capabilities, when examined closely, were effects derived from the viewpoints of nursing practices and human care [13] [14]. In this regard, robot therapy has not been generalized, and more descriptions and discussions about its practical utility are required.

A key challenge in dementia care is to assist the person sustain communication, connection, and environment with family and caregivers [15]. It was thought necessary to develop not only support of elderly people with dementia but also of robots assuming that families and nurses use such robots.

The aim of this study was to identify the characteristics of a transactive phenomenon in relationships between older adults with dementia and nurses as intermediaries, with communication robots.

\section{Method}

\subsection{Theoretical Framework}

The Transactive Relationship Theory of Nursing (TRETON) [16] [17] [18] explains the engagement processes that are characteristic activities among older adults with dementia, the nurse as mediator, and the communication robot. This theory explains the mutual engagement and the technological engagement that transpires among them. In testing the presence of these characteristics transactive relations, physiological determinants were used such as the wearable electrocardiography that measures heart rate variability indicating sympathetic and parasympathetic nervous activity. Together with video-recorded activities, the indications supporting the characteristics transactive phenomenon in relations among older adults with dementia, nurses, and communication robots are made evident.

\subsection{Procedure of Data Collection}

\subsubsection{Communication Robot with Remote-Controlled Speaker}

A smile supplement robot "Unazuki Kabochan $\odot$ (Nodding Kabochan, hereafter, Kabochan)" is a Cognitive Skill Aid Robot [19]. Nodding Kabochon can talk, sing, and slightly nod prettily in response to its owner's touch and spoken words. The Nodding Kabochan, is a 28-centimeter tall pumpkin-suited robot that affectionately interacts with older adults using its five sensors that are installed in its mouth, head, hands, feet, and main body. These sensors allow the Kabochan to 
verbally respond to any sounds and movements. Kabochan is pre-programmed to address its owner in eight different ways, including voicing words such as "Grandma" and "Grandpa". Moreover, the Kabochan contains several physical exercise modes, which includes a pose game, raising the flag game, as well as vocal singing exercises. In addition, a remote-controlled speaker, called Pechat $\odot$ [20] was attached to the Kabochan to enable a seamless mutual dialogue engagement. The Pechat can be programmed and allows the Kabochan to talk/sing by operating the dedicated "app". Pechat has a program function prepared by the nurse intermediaries (Figure 1).

\subsubsection{Older Persons with Dementia}

The two subjects of the study were older persons with dementia who have been diagnosed with dementia using Hasegawa's Dementia Scale-Revised (HDS-R) [21]. Two older persons a man and woman in their 80s participated in this study. Subject A: male, 80 's, person with dementia and his HDS-R score was 8 points. Subject B: female, 80 's, person with dementia and her HDS-R score was 10 points.

\subsubsection{Measuring Autonomic Nervous System Data}

The subjects were instructed to rest for 30 minutes before commencing with the intervention as transactive relations activities. The duration of the intervention was about 30 minutes using the Kabochan with Pechat, after which the exercise rehabilitation was performed by the subjects for 30 minutes. During the intervention time, the two nurses mediated with the Kabochan to enhance a smooth procedure-based transactive relationship. First, the conversation was between the Kabochan and the subjects. Next, one nurse operated the Pechat attached to the Kabochan, and the subjects interacted subsequently (Figure 2).

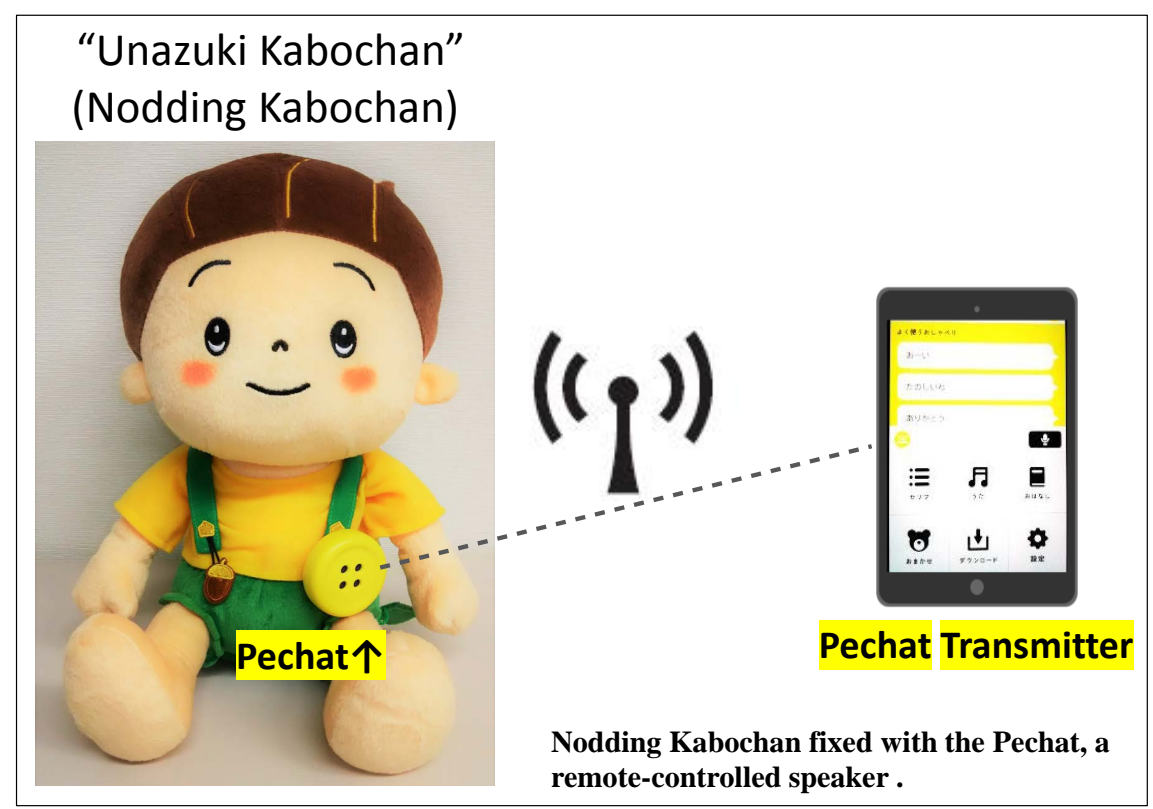

Figure 1. The Kabochan with pechat. 


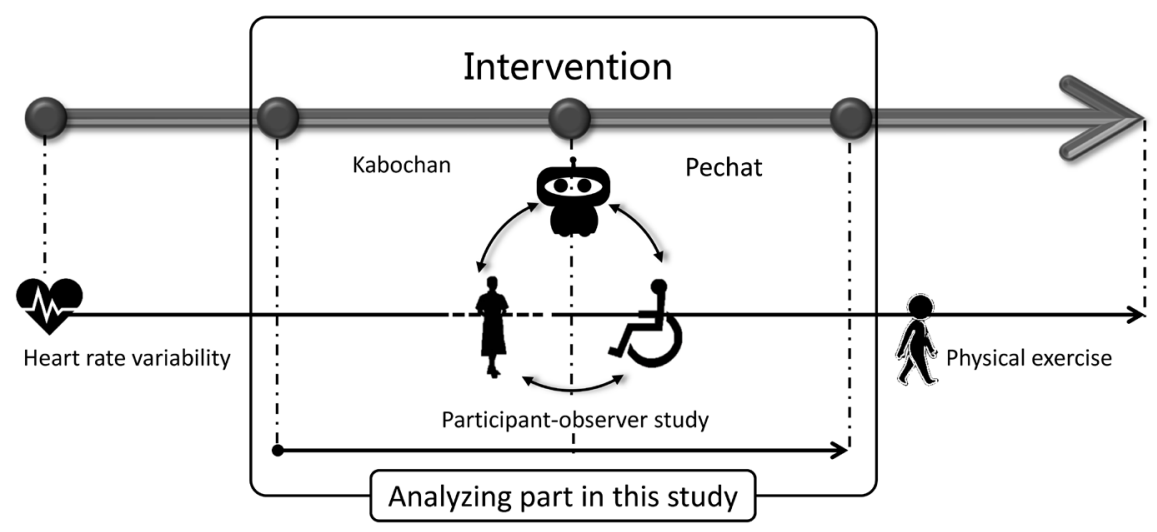

Figure 2. Intervention and analyzing part in this study.

\subsubsection{Analysis Methods of Autonomic Nervous Activity}

Heart Rate Variability (HRV) data were assessed at various frequency bands using an HRV software tool (MemCalc/win: GMS, Tokyo, Japan). In a continuously recorded data, interbeat (R-R) intervals were obtained for a 1-min segment using the maximum entropy method. In this study, the two major spectral components of HRV, the variances of the Low-Frequency (LF: $0.04-0.15 \mathrm{~Hz}$ ) band and High-Frequency (HF: $0.15-0.4 \mathrm{~Hz}$ ) band, were calculated. The HF data can be used as an index of parasympathetic nervous activity, and the LF/HF ratio can be used as an index of sympathetic nervous activity.

An optimal level of variability within an organism's key regulatory systems is critical to the inherent flexibility and adaptability or resilience that epitomizes healthy functioning and well-being [22]. These were suggested to determine the association between quality of emotional experience and the pattern reflected in the HRV waveforms [23].

$\mathrm{HRV}$ is the change in the time intervals between adjacent heartbeats. It is an emergent property of interdependent regulatory systems that operate on different time scales to adapt to environmental and psychological challenges [24]. The heart's rhythms are characterized as reflecting both physiological and psychological functional status of internal self-regulatory systems [24].

With psychological regulation, lower HF power is associated with stress, panic, anxiety, or worry [25]. Lowered parasympathetic activity, rather than reduced sympathetic functioning appears to account for the reduced HRV in aging [24]. However, a high LF/HF ratio may indicate higher sympathetic activity relative to parasympathetic activity. This can be observed when persons engage in meeting a challenge that requires effort and increased sympathetic activation. Alternatively, it can indicate increased parasympathetic activity as occurs during slow breathing [24]. In this study, the use of HRV is critical in measuring the psychological functional status and emotional experience of elderly persons particularly those with dementia.

\subsubsection{Analysis}

Changes in autonomic nervous activity were determined using HRV. Heart Rate (HR), HF, and LF/HF are shown in the graph, it shows the relationship between 
subjects' conversation with Kabochan and/or Kabochan with Pechat during intervention. The results were recorded graphically, enabling visual assessments and measurements.

\subsubsection{Data Collection Period}

Data collection period was December, 2016.

\subsubsection{Ethical Consideration}

The data collection procedure was performed following the Private Information Protection Law, with approval from the Tokushima University Hospital Ethics Board (approval number 2039) and Mifune Hospital (approval number 20170201-1). The purpose and methods used in the study were explained to all subjects and their guardians. Subjects were assured that their personal information were protected, that the report would be done as an aggregate, and will be used only for research purposes. Informed consents were obtained prior to the commencement of the study.

\section{Results}

The transactive relationship is presented as a conversational dialogue with older persons, the Kabochan or the Kabochan with Pechat, and two nurses. The results of the study are exhibited as a description of the activity of the autonomic nervous system measurements presented in Figures at specified data collection time.

Data collection was started in the morning. The conversational interaction with the Kabochan started at 10:38 a.m., and the conversation involving the Kabochan with Pechat was conducted from 10:50 until 11:09 a.m. At 10:38 a.m., Subject A touched the Kabochan's hands while saying "It is cute." Nurse A then moved the Kabochan to let Subject B see it. The Kabochan uttered the word "Heeeey!" while nodding. Subsequently, Subject A while in dialogue with Nurse $B$ made jokes and with facial expressions remarked, "I understand that Kabochan prefers a woman."

At this time, Subject A's parasympathetic nervous activity showed some marked elevation. It seemed to show that Subject A was having some health issues but seemed to respond well upon seeing the nodding of the Kabochan (Figure 3).

When Subject A looked at the state of Kabochan with the other subject communicating with it, the time subject's parasympathetic nervous activity increased.

At 10:44 a.m. The Kabochan began to show incoherent operating activities. This was near the end of the session. This was when Kabochan sang "Usagi to Kame: Rabbits and Turtles nursery rhymes" and uttered "I will forget the time" and "Wow, Thank you". These words signaled that the batteries needed to be replaced. Both the Kabochan and the nurses ceased to continue singing the song. However, as Subjects A and B seemed to be hard of hearing, they appeared not to hear that the Kabochan was not singing the song anymore.

As soon as the batteries were replaced, Kabochan began to sing again. Subject 
B clapped and Subject A nodded and sang the song together as if to teach the child. When the Kabochan stopped singing, the subjects applauded.

10:50 a.m. The Kabochan with Pechat uttered the question, "It is your birthday soon, isn't it?" Subject A responded with a question, "Why do you know my birthday. I am scared. Is someone checking me?” He seemed to show a sense of fear, as to how the Kabochan with Pechat knew his birthday. He then informed Nurse A that on hearing the Kabochan ask the question, he felt "a shiver up his spine" while at the same time showing a smile. At this time, Subject A's parasympathetic nervous activity increased from 25.09 to $325.77 \mathrm{msec}^{2}$ (Figure 4).
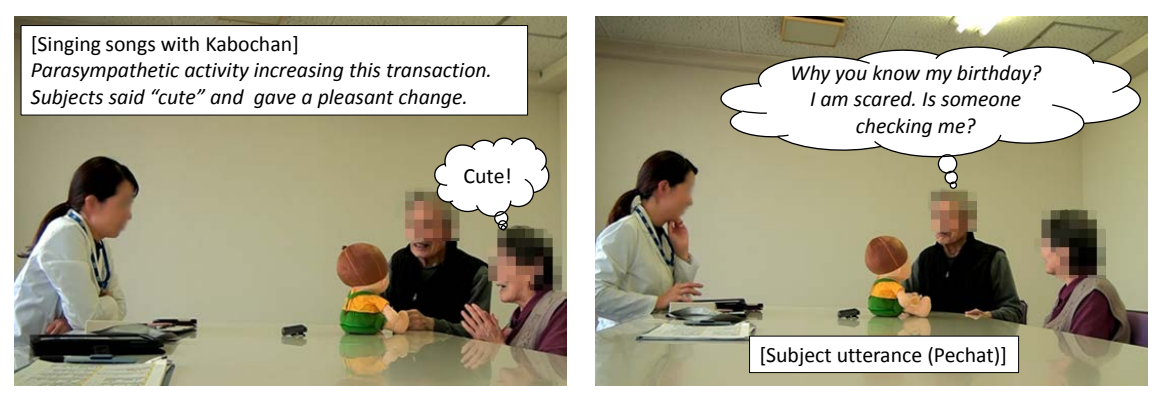

Figure 3. Transactive Relationship between the nurse, Kabochan, and the older adults with dementia.

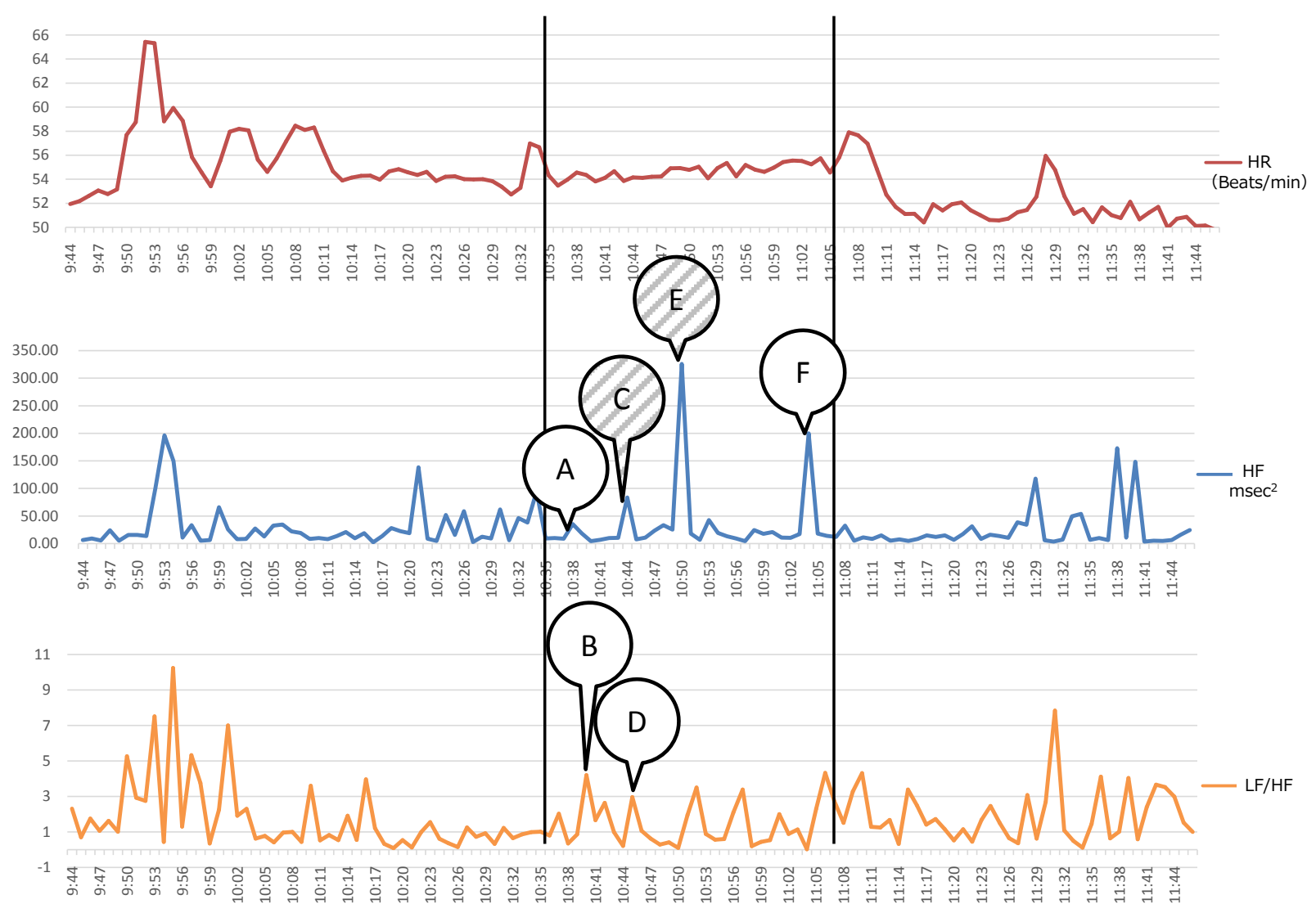

Figure 4. HR, HF, LF/HF and the relationship between subjects' conversation with Kabochan and/or Kabochan with Pechat during intervention (Subject A). 
The alphabet in the figure shows the relationship between heart rate variability of the older adults with dementia and nurse, Kabochan or Kabochan with Pechat.

A) 10:38. Looking at the situation that Kabochan faces the B and says "Heeey!" with nodding, the Subject speaks to the nurse that Kabochan prefers the woman.

B) 10:40. During the song is singing, Kabochan suddenly nodded.

C) 10:44. Sing a song "Moshimoshi kameyo"

D) 10:45. Subject A said I want to take this Kabochan to my home. That's cute!

E) 10:50. "Why does Kabochan know my birthday? I am scared. Is someone cheking me?"

F) 11:04. By listening to the Nurse and Subject B, Kabochan with Pechat is calling Subject $A$ 's first name, $M r$. A.

At 10:36 a.m. Nurse B showed what the Kabochan's reactions was by touching and talking to it. Subject B said "It's cute."

At 10:45 a.m. Talking with the Kabochan, Subject A remarked "This Kabochan is clever" "Yes, he looks good and have good facial expression."

At 10:55 a.m. In responding to the Kabochan's question, "Where do you want to go?" Subject B explained to Nurse A and B about the mountain where she wanted to go.

At 10:59 a.m. Subject B listened to the Nurses calling on the Kabochan with Pechatas to whether it can say the name of Subject A.

At 11:04 a.m. Explaining that the Kabochan with Pechat is calling the name of Subject A while listening to Nurse A and Subject B, the Kabochan with Pechat called Subject A's first name. That's when Subject A's parasympathetic activity increased.

At 11:07 a.m. While talking to subject A, subject B asked the Kabochan with Pechat, "You're with me, right?" (Figure 5 and Figure 6).

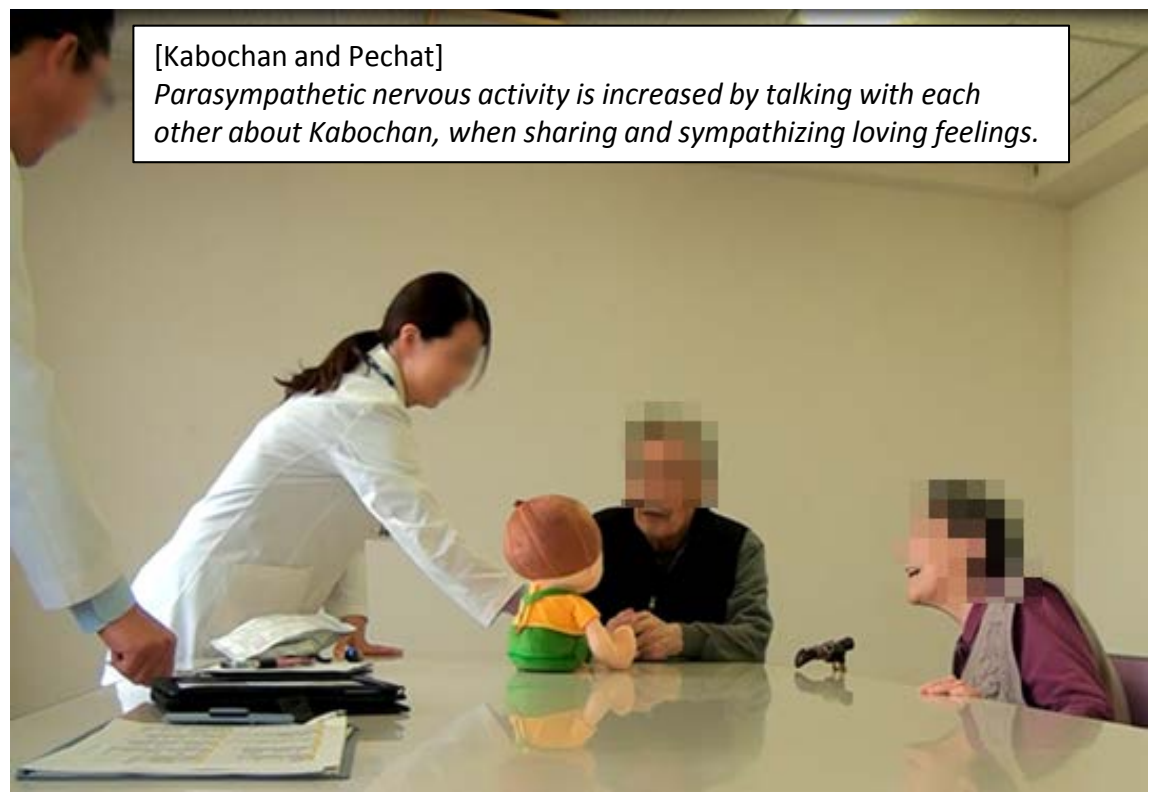

Figure 5. Expressions of transactive relationship between the nurse, Kabochan with Pechat, and the older adults with dementia. 


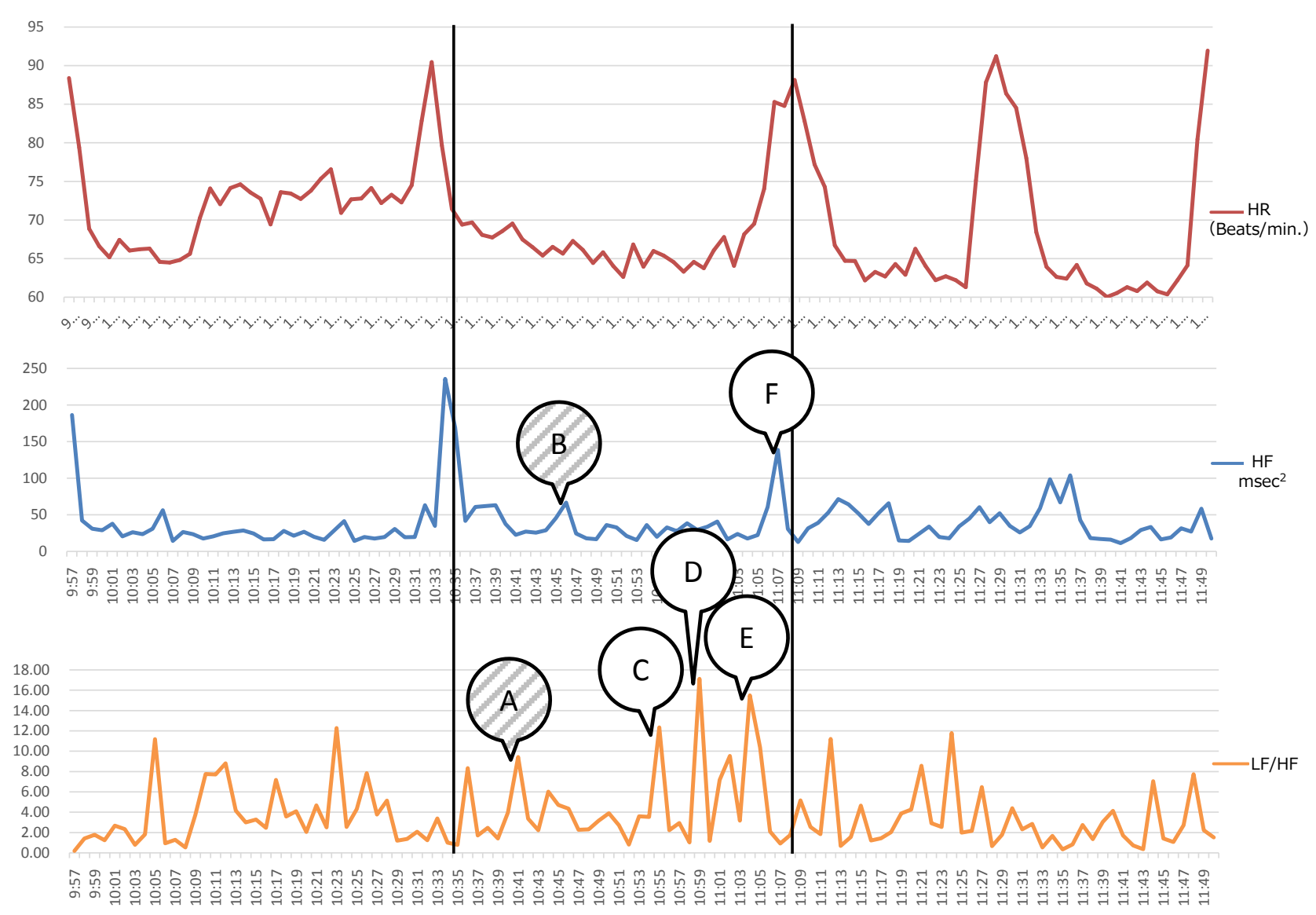

Figure 6. HR, HF, LF/HF and the relationship between subjects' conversation with Kabochan and/or Kabochan withPechat during intervention (Subject B).

The alphabet in the figure shows the relationship between heart rate variability of the older adults with dementia and nurse, Kabochan or Kabochan with Pechat.
A) 10:36. Nurse B showed how Kabochan reacts by touching and talking to. Subject B said "It's cute."
B) 10:45. Talking with Subject A as "This Kabochan is clever", "Yes, looks good face".
C) 10:55. In response to the question of Kabochan's "Where do you want to go?" Subject $B$ explained about mountain where she wants to go to there.
D) 10:59. Subject B listens to for the Nurse calling on whether Kabochan with Pechat can say the name of Subject $A$.
E) 11:04. Teaching that Kabochan with Pechat is calling the name of Subject A.
F) 11:07. While talking to subject A, subject B ask to Kabochan with Pechat, "You're with me, right?"

At 10:38 a.m. Subject B asked, "Please let me know your name?" But since the Kabochan cannot answer, instead it said "I do not know." That's when Nurse A and B managed the "Kabochan" to smile.

At 10.39 a.m. As Subject B wanted to know how old Kabochan was, Nurse A asked the Kabochan several times: "How old are you?" However, the Kabochan did not respond appropriately and instead replied "Wow, I will forget my time, 
please replace my battery." Because the Kabochan did not respond well, Nurse B switched to Kabochan with Pechat mode, and the nurses decided to sing a song together (with the Kabochan with Pechat). Subject A has seen the exchange between and among the Kabochan, Subject B, Nurse A and B with a gentle look (see Figure 7).

\section{Discussion}

As the demands and needs rise for quality health care particularly in the older adult population, the utilization of human-machine process requirements have intensified and the realities and consequences of transactive relationships have become integral to assuring quality human health care. An outstanding result of the study was the patient singing the song together the Kabochan with Pechat. In explaining the characteristic transactive relationships, the evidence using physiological instrumentations and video-recorded images supports the recognition of opportunities for distinguishing transactive relations which are valuable to the effective application of communication robots in human health care situations, especially for older adults with dementia. Three thematic situations were revealed, namely, a sense of fear, a pleasant sensation, and misunderstanding and confusion.

\subsection{Situation 1: A Sense of Fear}

When the Kabochan with Pechat uttered the question, "It is your birthday soon, isn't it?" Subject A said, "Why do you know my birthday. I am scared." "Is someone checking me?" Subject A felt a sense of fear as to how the Kabochan with Pechat knew his birthday. He then informed Nurse A that he had a heightened sensation up his spine while at same time showing a smile. At that time, his parasympathetic nervous activity increased from 25.09 to $325.77 \mathrm{msec}^{2}$.

Anxiety is one of the major symptoms of dementia and is often exhibited as some form of fear reaction. Subject A showed some facial expression of fear that was not related to dementia, but rather seemed associated with the realization that his personal secret is known by someone. However, older adults with dementia are easily confused. Attention should be made regarding situations or occasions causing some kind of "fear" among older persons with dementia. As nurse intermediaries, it is critical that meticulous attention be considered when
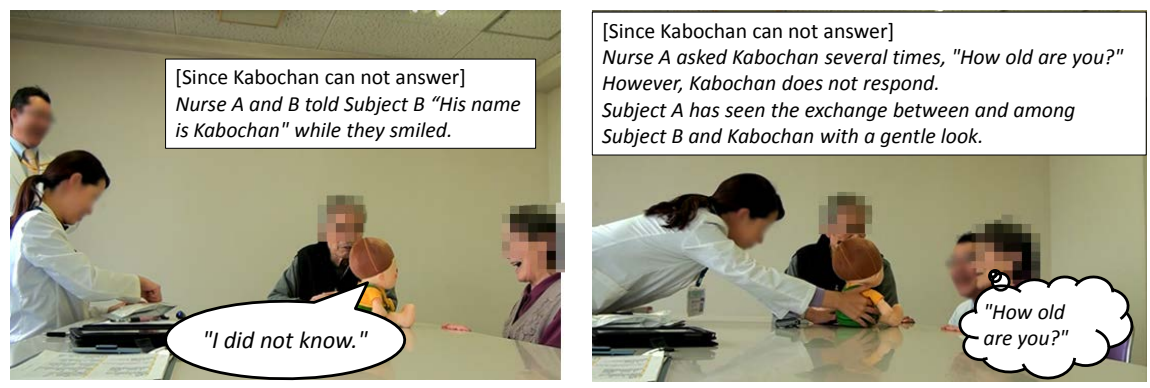

Figure 7. Transactive relationship between the nurse, Kabochan with Pechat, and the elderly patients with dementia. 
using personal information reflecting memory evidence as information (Figure 3).

\subsection{Situation 2: A Pleasant Sensation}

When Subject B clapped, and Subject A nodded and sang a song together with the Kabochan with Pechat, the situation seemed to illustrate that both were teaching a child to sing. When the singing was over, they applauded. The situation seemed to show that at that time, while singing songs with the Kabochan, the subjects' parasympathetic activity increased and gave a pleasant "social" change to the subjects' personality as evidenced by their facial expressions (Figure 4).

Sakamoto et al. [25], reported that passive and interactive music interventions for individuals with severe Alzheimer's disease caused short-term parasympathetic dominance. Music intervention, such as singing popular tunes, can reduce stress in individuals with severe dementia, with interactive interventions exhibiting the strongest beneficial effects. Since interactive music intervention can restore residual cognitive and emotional function, such approach may be useful for aiding severe dementia patients' relationships with others and improving QOL.

\subsection{Situation 3: Misunderstanding and Confusion}

In another scene, Subject B asked, "Please let me know your name?" But since the Kabochan cannot answer the question correctly and instead said "I did not know", Subject B seemed confused. The Kabochan was not programmed to respond to other questions. At this time, the limited capacity for interaction between the Kabochan, the nurses, and the subjects, was disadvantageous to the subjects' reality and in determining the transactive characteristic features (Figure 7).

However, as Subjects A, and B seemed to be hard of hearing, they may not have heard the incoherent response. Unfortunately, the nurses noticed that the Kabochan was not functioning well. Therefore, to prevent the subjects from experiencing confusing transactive responses through the Kabochan's incorrect responses, the nurses as intermediaries intervened by conducting a smooth and pleasant conversation with the subjects. This was fulfilling one of the roles of an expert nurse coordinator. Nurse A and B told Subjects A and B while smiling, that "His name is Kabochan." Subject A saw the exchange among Subject B, Nurse A and the Kabochan and expressed a gentle smile.

Prior to this occasion, Subject B found the Kabochan to be "cute." This was the expression when Subject B heard the Kabochan's voice. The voice of the Kabochan was of a high-pitched frequency range, like that of a boy child. Although it was possible to program the vocalization of the Kabochan to be low-pitched, in doing so, the "cuteness" of Kabochan may be lost.

With age-related hearing loss, or presbycusis, the slow loss of hearing that occurs as people get older, it is characterized by reduced hearing sensitivity and 
speech understanding in noisy environments, slowed central processing of acoustic information, and impaired localization of sound sources [26]. It is characterized by a decline in auditory functioning, such as increased hearing thresholds and poor frequency resolution [27]. As the voice of the Kabochan with Pechat was relatively high-pitched, an unnatural electronic quality, it seemed that there were times when it was difficult for the older adults with dementia to hear it. Countermeasures against this situation include modulations in communication robot voice sound frequency, adjustments in speed of verbalizations, and increase in the amount of information given at one time through the lengthening of the utterances of the communication robot [28]. It is also critically necessary to take appropriate measures to use the communication robot display so that the elderly can understand the voice of the communication robot and the conversation contents will be useful rather than confusing.

Inoue et al. pointed out that the timing for robots making a smooth speaking voice is difficult [29]. Therefore, it may be assumed that with the participation of nurses as intermediaries, the mutual engagement through conversation among communication robot and older adults with dementia will be of greater usefulness as intervention in elderly care, particularly for patients with dementia.

\subsection{Implications for Practice}

In this research, the transactive relations among the robot (Kabochan and Pechat), older adults with dementia and nurses as intermediaries revealed the characteristics of the transactive relationships. If communication robots can enhance communicative interaction of older adults with dementia, one of the main challenges is developing a means for an effective and intuitive human-robot interaction. It was suggested that communication robots can keep its voice at a comfortable tone and pitch and its volume can be adjusted as it vocalizes to utter words.

While humans can express their intentions in different ways (e.g., physical gestures or motions, or speech or language patterns, with robots, gaze-based implicit intention) robots are limited as communication programming is still developing [30].

However, the hope is, if this function for robots is developed well, the conversation between the elderly and the communication robot will be much smoother and characteristic transactive relationships can be easily identified, organized and used to enhance programming of future humanoid nurse robots [16] [17] [18].

When developing future communication robots for the elderly with dementia, it is considered that an effective conversation will occur best, when this communication robot development includes characteristic transactive relations with nurses as intermediaries. The role functions of these intermediaries have been shown to be of high versatility, indicating that situations such as these may also be controlled by other health care providers such as by occupational therapists in their respective practices.

\subsection{Limitations of This Study}

Limitation of this study was conducted using HRV and the qualitative word de- 
scriptions of two elderly patients during the transactive relationship process between a communication robot and the two nurses. The capability of the HRV to determine neurophysiological response mechanisms determined the predictive effects of the transactive value of the Communication Robot, thereby providing some procedural evidence for using HRV. The influence of robotic technology as therapy suggested a positive and significant outcome. It is thereby envisioned that a study can be conducted to focus on the effect of robot-human interaction using Communication Robots in the future. Using HRV and other parameters which can measure the cognitive status of elderly persons particularly those with dementia is possible.

\section{Conclusions}

This study identified the characteristics of the transactive phenomenon in relationships among older adults with dementia, with nurses as intermediaries and the communication robot, that is, the Kabochan with Pechat. These characteristic transactive relationships include changes in autonomic nervous system activity of older adults with dementia that are measureable every minute, the linguistic responses of elderly people, and the interactive consequences between the communication robots and nurses as exhibited in the appropriate Figures. These transactive relationships are described in three unique situations, namely: $A$ Sense of Fear, A Pleasant Sensation, and Misunderstanding and Confusion. The electrographic data (Figure 4 and Figure 6) provided neurophysiological evidence of the influence of robot utilization on the autonomic nervous system activity of older adults with dementia.

However, communication robots have continuing issues pertaining to its capabilities to express competence in its actions. Nevertheless, emotional conversations can be established and improved by controlling the communication robot activities with a remote-controlled speaker. Furthermore, the study also revealed that when communication robots with remote-controlled speaker vocalize information about the patient's personal information in a conversation, the patient feels a sense of fear. Autonomic nervous system data supports this emotional effect when communication robots are able to converse, even if the dialogue is time-limited.

\section{Acknowledgements}

We would like to express our deep gratitude to the subjects/participants of this study. In particular, we wish to thank the following clinicians for their participation in this study: Dr. Kazushi Mifune, MD; PhD, Dr. Hiroshi Ogasawara, MD; PhD, and Ms. Keiko Nagao, RN, Head Nurse (San Ai Kai). This work was partly supported by JSPS KAKENHI Grant Number JP 24390477, and partly supported by Grant of the Tokushima University.

\section{References}

[1] Statistics Bureau Portal Site of Official Statistics of Japan. (In Japanese) 
http://www.stat.go.jp/data/jinsui/ www.stat.go.jp/data/nenkan/65nenkan/zuhyou/y650202000.xls

[2] Siegel, C. and Dorner, T.E. (2017) Information Technologies for Active and Assisted Living-Influences to the Quality of Life of an Ageing Society. International Journal of Medical Informatics, 100, 32-45. https://doi.org/10.1016/j.ijmedinf.2017.01.012

[3] Ministry of Health, Labour and Welfare in Japan (2016) Long-Term Care Insurance System of Japan.

http://www.mhlw.go.jp/english/policy/care-welfare/care-welfare-elderly/dl/ltcisj_e. pdf

[4] New Energy and Industrial Technology Development Organization: Robotic Solutions; Building a Smart Society with State-of-the-Art Technology. http://www.nedo.go.jp/content/100582006.pdf

[5] Taketomi, T. and Sankai, Y. (2012) Walking Assistance for Cerebral Palsy with Robot Suit HAL. Japanese Society for Medical and Biological Engineering, 50, 105-110.

[6] Ohnuma, T., Lee, G. and Chong, N.Y. (2017) Development of JARoW-II Active Robotic Walker Reflecting Pelvic Movements While Walking. Intelligent Service Robotics, 10, 95-107. https://doi.org/10.1007/s11370-016-0212-7

[7] Takei, M., Chikami, S., Iida, T., Saita, R., Kubomura, C. and Kamada, H. (2008) Design and Implementation of a Prototype of Dialogue Robot with a Personal Character. IEICE Technical Report, 108, 13-18.

[8] Shiba, H., Kanoh, M., Kato, S., Kunitachi, T. and Itoh, H. (2005) A Facial Control Method Using Emotional Parameters in Sensibility Robot. IEEJ Transactions on Electronics, Information and Systems, 125, 1852-1860. https://doi.org/10.1541/ieejeiss.125.1852

[9] Schwiegelshohn, F., Wehner, P., Rettkowski, J., Göhringer, D., Hübner, M., Keramidas, G., Antonopoulos, C. and Voros, N.S. (2015) A Holistic Approach for Advancing Robots in Ambient Assisted Living Environments. 2015 IEEE 13 th International Conference on Embedded and Ubiquitous Computing, Porto, 21-23 October 2015, 140-147. https://doi.org/10.1109/EUC.2015.37

[10] Bernabei, V., De Ronchi, D., La Ferla, T., Moretti, F., Tonelli, L., Ferrari, B., Forlani, M. and Atti, A.R. (2013) Animal-Assisted Interventions for Elderly Patients Affected by Dementia or Psychiatric Disorders: A Review. Journal of Psychiatric Research, 47, 762-773. https://doi.org/10.1016/j.jpsychires.2012.12.014

[11] Van Wynsberghe, A. (2013) Designing Robots for Care: Care Centered Value-Sensitive Design. Science and Engineering Ethics, 19, 407-433.

https://doi.org/10.1007/s11948-011-9343-6

[12] Tanaka, M., Ishii, A., Yamano, E., et al. (2012) Effect of a Human-Type Communication Robot on Cognitive Function in Elderly Women Living Alone. Medical Science Monitor, 18, CR550-CR557.

[13] Kuwamura, K., Nishio, S. and Sato, S. (2016) Can We Talk through a Robot as If Face-to-Face? Long-Term Fieldwork Using Teleoperated Robot for Seniors with Alzheimer's Disease. Frontiers in Psychology, 7, 1066.

https://doi.org/10.3389/fpsyg.2016.01066

[14] Pino, M., Boulay, M., Jouen, F. and Rigaud, A.-S. (2015) Are We Ready for Robots That Care for Us? Attitudes and Opinions of Older Adults toward Socially Assistive Robots. Frontiers in Aging Neuroscience, 7, 141. https://doi.org/10.3389/fnagi.2015.00141

[15] Mordoch, E., Osterreicher, A., Guse, L., Roger, K. and Thompson, G. (2012) Use of Social Commitment Robots in the Care of Elderly People with Dementia: A Litera- 
ture Review. Maturitas, 74, 14-20. https://doi.org/10.1016/j.maturitas.2012.10.015

[16] Tanioka, T. (2017) The Development of the Transactive Relationship Theory of Nursing (TRETON): A Nursing Engagement Model for Persons and Humanoid Nursing Robots. Int J Nurs Clin Pract, 4, 223. https://doi.org/10.15344/2394-4978/2017/223

[17] Tanioka, T. (2017) The Transactive Relationship Theory of Nursing. In: Tanioka, T., Yasuhara, Y., Osaka, K., Ito, H. and Locsin, R., Eds., Nursing Robots. Robotic Technology and Human Caring for the Elderly, Fukuro Shuppan Publishing, Okayama, Japan.

[18] Tanioka, T., Osaka, K., Locsin, R., Yasuhara, Y. and Ito, H. (2017) Recommended Design and Direction of Development for Humanoid Nurse Robots Perspective from Nursing Researchers. Intelligent Control and Automation.

[19] Nodding Kabochan: Cognitive Skill Aid Robot, By: Youjin Kang, Intern, AARP International.

http://www.aarpinternational.org/resource-library/resources/nodding-kabochan-co gnitive-skill-aid-robo

[20] https://pechat.jp/

[21] Imai, Y. and Hasegawa, K. (1994) The Revised Hasegawa's Dementia Scale (HDSR)-Evaluation of Its Usefulness as a Screening Test for Dementia. Hong Kong Journal of Psychiatry, 4, 20-24.

[22] Huang, Q. and Tang, J. (2010) Age-Related Hearing Loss or Presbycusis. European Archives of Oto-Rhino-Laryngology, 267, 1179-1191.

https://doi.org/10.1007/s00405-010-1270-7

[23] Shaffer, F., McCraty, R. and Zerr, C.L. (2014) A Healthy Heart Is Not a Metronome: An Integrative Review of the Heart's Anatomy and Heart Rate Variability. Frontiers in Psychology, 5, 1040. https://doi.org/10.3389/fpsyg.2014.01040

[24] McCraty, R., Atkinson, M., Tomasino, D. and Bradley, R.T. (2009) The Coherent Heart: Heart-Brain Interactions, Psychophysiological Coherence, and the Emergence of System-Wide Order. Integral Review, 5, 10-115.

[25] McCraty, R. and Shaffer, F. (2015) Heart Rate Variability: New Perspectives on Physiological Mechanisms, Assessment of Self-regulatory Capacity, and Health Risk. Global Advances in Health and Medicine, 4, 46-61.

[26] Sakamoto, M., Ando, H. and Tsutou, A. (2003) Comparing the Effects of Different Individualized Music Interventions for Elderly Individuals with Severe Dementia. International Psychogeriatrics, 25, 775-784.

[27] Yamasoba, T., Lin, F.R., Someya, S., Kashio, A., Sakamoto, T. and Kondo, K. (2013) Current Concepts in Age-Related Hearing Loss: Epidemiology and Mechanistic Pathways. Hearing Research, 303, 30-38.

[28] Kanoh, M., Oida, Y., Nomura, Y., Araki, A., Konagaya, Y., Ihara, K., Shimizu, T. and Kimura, K. (2011) Examination of Practicability of Communication Robot-Assisted Activity Program for Elderly People. JRM, 23, 3-12. https://doi.org/10.20965/jrm.2011.p0003

[29] Inoue, K., Sasaki, C. and Nakamura, M. (2015) Communication Robots for Elderly People and Their Families to Support Their Daily Lives-Case Study of Two Families Living with the Communication Robot. Studies in Health Technology and Informatics, 217, 980-983.

[30] Li, S. and Zhang, X. (2017) Implicit Intention Communication in Human-Robot Interaction through Visual Behavior Studies. IEEE Transactions on Human-Machine Systems, 1-12. https://doi.org/10.1109/thms.2017.2647882 
Submit or recommend next manuscript to SCIRP and we will provide best service for you:

Accepting pre-submission inquiries through Email, Facebook, LinkedIn, Twitter, etc. A wide selection of journals (inclusive of 9 subjects, more than 200 journals)

Providing 24-hour high-quality service

User-friendly online submission system

Fair and swift peer-review system

Efficient typesetting and proofreading procedure

Display of the result of downloads and visits, as well as the number of cited articles Maximum dissemination of your research work

Submit your manuscript at: http://papersubmission.scirp.org/

Or contact ica@scirp.org 